JMB

\title{
Structure of the NCoA-1/SRC-1 PAS-B Domain Bound to the LXXLL Motif of the STAT6 Transactivation Domain
}

\author{
Adelia Razeto', Venkatesh Ramakrishnan', Claudia M. Litterst ${ }^{2}$ \\ Karin Giller ${ }^{1}$, Christian Griesinger ${ }^{1}$, Teresa Carlomagno ${ }^{1}$ \\ Nils Lakomek ${ }^{1}$, Thomas Heimburg ${ }^{3}$, Marco Lodrini ${ }^{2}$, Edith Pfitzner ${ }^{2}$ and \\ Stefan Becker ${ }^{1 *}$
}

\author{
${ }^{1}$ Department for NMR-based \\ Structural Biology \\ Max-Planck-Institute for \\ Biophysical Chemistry, Am \\ Faßberg 11, 37077 Göttingen \\ Germany
}

${ }^{2}$ Georg-Speyer-Haus, Institute for Biomedical Research Paul-Ehrlich-Straße 42-44 60596 Frankfurt, Germany

${ }^{3}$ Membrane Biophysics and Thermodynamics Group Max-Planck-Institute for Biophysical Chemistry, Am Faßberg 11, 37077 Göttingen Germany

\begin{abstract}
Signal transducer and activator of transcription 6 (STAT6) regulates transcriptional activation in response to interleukin- 4 (IL-4) by direct interaction with coactivators. The CREB-binding protein (p300/CBP) and the nuclear coactivator 1 (NCoA-1), a member of the p160/steroid receptor coactivator family, bind independently to specific regions of the STAT6 transactivation domain and act as coactivators. The interaction between STAT6 and NCoA-1 is mediated by an LXXLL motif in the transactivation domain of STAT6. To define the mechanism of coactivator recognition, we determined the crystal structure of the NCoA-1 PAS-B domain in complex with the STAT6 LXXLL motif. The amphipathic, $\alpha$-helical STAT6 LXXLL motif binds mostly through specific hydrophobic interactions to NCoA-1. A single amino acid of the NCoA-1 PAS-B domain establishes hydrophilic interactions with the STAT6 peptide. STAT6 interacts only with the PAS-B domain of NCoA- 1 but not with the homologous regions of NCoA-2 and NCoA-3. The residues involved in binding the STAT6 peptide are strongly conserved between the different NCoA family members. Therefore surface complementarity between the hydrophobic faces of the STAT6 fragment and of the NCoA-1 PAS-B domain almost exclusively defines the binding specificity between the two proteins.
\end{abstract}

(C) 2003 Elsevier Ltd. All rights reserved.

Keywords: NCoA-1; STAT6; PAS-B domain; transactivation domain; LXXLL motif

\section{Introduction}

STAT proteins mediate signaling from activated cytokine receptors to the nucleus. ${ }^{1}$ Following phosphorylation at a specific tyrosine residue by receptor associated Janus kinases, STATs form homo- or heterodimers and translocate into the nucleus. ${ }^{2}$ They bind to specific DNA sequences and regulate the transcription of target genes by direct interaction with components of the transcription

Supplementary data associated with this article can be found at doi: 10.1016/S0022-2836(03)01568-7

Abbreviations used: SRC, steroid receptor coactivator; PAS, Per-Arnt-Sim.

E-mail address of the corresponding author: sabe@nmr.mpibpc.mpg.de machinery or via coactivators that act as bridging factors and modifiers of the chromatin structure. ${ }^{2-4}$

STAT6 activation is triggered by the interleukins IL-4 and IL-13. ${ }^{5}$ It almost exclusively mediates the expression of genes activated by these cytokines. IL-4 signaling regulates the expression of genes involved in immune and anti-inflammatory responses. STAT6 knockout mice lack the IL-4mediated Th2 development and immunoglobulin class switching to the IgE isotype. ${ }^{6,7}$

The C-terminal part of the STATs constitutes the transactivation domain. In all STATs the transactivation domain is relatively acidic and prolinerich, ${ }^{1,8}$ resulting in high structural flexibility. Crystal structures of STATs were only obtained with constructs lacking the transactivation domain. ${ }^{9,10}$ Therefore this domain is structurally not very well characterized. STAT6 has an 
extended transactivation domain, which does not have homologues in other STATs. It was characterized as a modular region ${ }^{11,12}$ with two distinct transactivation functions. ${ }^{13}$

By direct interaction with specific parts of its transactivation domain, STAT6 recruits the coactivators p300/CBP and NCoA-1, which are required for transcriptional activation by IL-4. ${ }^{14,15}$ p300/CBP form a multisubunit complex with other coactivators like the NCoA coactivator family and $\mathrm{p} / \mathrm{CAF}^{16}$ This multicomponent coactivator complex interacts with many DNA-binding transcriptional activators, like nuclear receptors and other signal-regulated activators. ${ }^{17}$ The family of the NCoA coactivators, also called the p160/steroid receptor coactivator (SRC), currently consists of three homologous proteins, NCoA-1/SRC1, NCoA-2/TIF2/GRIP1 and NCoA-3/p/CIP/ACTR/AIB. ${ }^{18,19}$

Transcriptional activators function in concert with diverse coactivator proteins. It has been proposed that distinct classes of transcription factors selectively use specific coactivators with distinct histone acetyltransferase activities. ${ }^{20,21}$ In accordance with this model, a specific role of NCoA-1, but not NCoA-2 and NCoA-3, for STAT6-mediated transactivation was observed. ${ }^{14}$

STAT6 directly contacts NCoA-1 via an LXXLL interaction motif in the carboxyl-terminal part of its transactivation domain. ${ }^{22} \mathrm{~A}$ region between amino acid residues $213-462$ in NCoA-1, which comprises the region $\mathrm{B}$ of a predicted Per-ArntSim (PAS) domain, ${ }^{23}$ mediates the interaction with STAT6. $^{22}$ NCoA-1 is an essential coactivator for STAT6 transactivation as shown by overexpression of the STAT6-interacting domain of NCoA-1, which acts in a dominant negative manner. ${ }^{14}$ In addition, a STAT6 mutant lacking the LXXLL motif was strongly compromised in its potential to transactivate IL-4 responsive target genes. ${ }^{22} \mathrm{~A}$ synthetic peptide, which includes the STAT6 LXXLL motif as well as antibodies raised against this peptide totally abolished the STAT6/NCoA-1 interaction. Thus the LXXLL motif of STAT6 is the only portion of STAT6 that contacts NCoA-1.

In order to investigate the molecular mechanism of transcriptional stimulation by coactivators, we studied the structural features of NCoA-1 recognition by STAT6. Here we report the first structure of a coactivator PAS domain in complex with another protein.

\section{Results}

\section{Structure determination}

The NCoA-1 domain that interacts with STAT6 had been mapped to amino acid residues 257-420 by GST-pulldown experiments (data not shown). To form the complex, a synthetic peptide containing amino acid residues 794-814 of STAT6 was combined with the recombinant NCoA-1 fragment
257-420 and loaded on a Superdex75HR gel filtration column. MALDI-MS analysis from the elution peaks demonstrated, that the NCoA-1(257-420) fragment co-migrated with the STAT6(794-814) peptide. Initial trials to crystallize this complex failed. Secondary structure prediction with the program SOPMA $^{24}$ indicated that the amino acid residues located C-terminal of Asp366 were forming a coil. The affinity of the 257-370 NCoA-1 fragment for the STAT6(794-814) peptide interaction was investigated by isothermal titration calorimetry (ITC). A dissociation constant $\left(K_{\mathrm{D}}\right)$ of $0.8 \mu \mathrm{M}$ was obtained assuming a 1:1 binding model (see Supplementary Material). Through limited proteolysis a fragment spanning amino acid residues 257-385 was identified (see Supplementary Material). Crystals obtained from the NCoA-1(257-385)/STAT6(794-814) complex were suitable for X-ray analysis. They diffracted to $2.2 \AA$ resolution on a laboratory X-ray source.

The structure of the complex was determined by single anomalous dispersion (SAD) from non-covalently bound iodide ions ${ }^{25}$ and refined up to a $R$ free of $21.4 \%$ and a $R$-factor, calculated with all reflections, of $17.0 \%$ (Table 1 ). There are no outliers in the Ramachandran plot, as calculated by PRO$\mathrm{CHECK}^{26}$ (Table 1). The coordinate error estimated from the diffraction-component precision index ${ }^{27}$ is 0.20 . The model of the complex between NCoA-1(257-385) and STAT6 (794-814) consists of residues 259-367 from NCoA-1 and residues 795-808 from STAT6, with a total number of 119 amino acid residues: amino acid residues 350-353 of the NCoA-1 fragment are disordered. Solution state NMR revealed that the C-terminal residues from Leu371 show very sharp ${ }^{15} \mathrm{~N}$ as well as ${ }^{1} \mathrm{H}$ lines indicative of a highly flexible C-terminal part (data not shown). This observation confirms the domain limit observed in the crystal structure. According to mass spectrometry the crystals contain all the 153 residues of the complex: 132 amino acid residues of NCoA-1(257-385) with three additional $\mathrm{N}$-terminal residues due to the fusion protein construct and 21 amino acid residues of the STAT6(794-814) fragment, confirming its integrity. Thus the residues missing from the model are disordered in the crystal.

\section{The NCoA-1(259-367) domain is a canonical PAS domain}

In agreement with structural predictions, ${ }^{23}$ the NCoA-1(259-367) fragment forms a PAS domain. We call it NCoA-1 PAS-B domain, as upstream an additional PAS domain is predicted. The NCoA-1 PAS-B domain consists of a five-stranded anti-parallel $\beta$-sheet $\left(A_{\beta}\right.$ to $\left.H_{\beta}\right)$ and three $\alpha$-helices, $C_{\alpha}$ to $E_{\alpha}$, which connect the second and third $\beta$-strands, $\mathrm{B}_{\beta}$ and $\mathrm{F}_{\beta}$ (Figure 1). The STAT6 peptide binds between helix $D_{\alpha}$ and strand $B_{\beta}$ of the NCoA-1 PAS-B domain. Residues 795-798 are in extended conformation, while the residues $799-807$ form a two and a half turns $\alpha$-helix. The axis of the 
Table 1. X-ray data collection, phasing and refinement statistics

\begin{tabular}{|c|c|}
\hline \multicolumn{2}{|l|}{ Data collection } \\
\hline $\begin{array}{l}\text { Resolution range: overall/outer } \\
\text { shell }(\AA)\end{array}$ & $19.2-2.21 / 2.25-2.21$ \\
\hline Space group/unit cell $(\AA)$ & $\begin{array}{c}P 6_{2} / a=62.1, b=62.1, c= \\
73.4\end{array}$ \\
\hline Reflections: measured/unique & $137,915 / 8138$ \\
\hline Completeness ${ }^{\mathrm{a}}$ overall/outer shell & 99.7/98.1 \\
\hline Redundancy ${ }^{\mathrm{a}}$ overall/outer shell & $16.8 / 16.0$ \\
\hline$\langle I / \sigma(I)\rangle^{\mathbf{a}}$ overall/outer shell & $29.9 / 8.7$ \\
\hline$R_{\text {merge }}, \mathrm{a} \mathrm{b}$ overall/outer shell & $0.089 / 0.325$ \\
\hline \multicolumn{2}{|l|}{ Phasing } \\
\hline Anomalous $|\Delta F| / \sigma(\Delta F)$ in $3.2-3.0 \AA$ & 4.85 \\
\hline Best $\mathrm{CC} / \mathrm{CC}_{\text {weak }}(\%)$ & $37.6 / 20.2$ \\
\hline $\begin{array}{l}\text { Final weighted contrast (enantio- } \\
\text { morph) }\end{array}$ & $0.643(0.050)$ \\
\hline Connectivity (enantiomorph) & $0.922(0.718)$ \\
\hline \multicolumn{2}{|l|}{ Refinement } \\
\hline No. of residues protein/peptide & $105 / 14$ \\
\hline $\begin{array}{l}\text { No. of water molecules/iodide } \\
\text { ions }\end{array}$ & $116 / 5$ \\
\hline$R$-factor $/ R$-free ${ }^{\mathrm{c}}$ & $0.170 / 0.214$ \\
\hline r.m.s.d. bond lengths $(\AA)$ & 0.011 \\
\hline r.m.s.d. bond angles (deg.) & 1.394 \\
\hline \multicolumn{2}{|l|}{ Average $B$-factor $\left(\AA^{2}\right)$} \\
\hline Protein/peptide & $37.3 / 41.4$ \\
\hline Water molecules & 49.4 \\
\hline Iodide ions & 33.5 \\
\hline \multicolumn{2}{|l|}{ Ramachandran plot (\%) } \\
\hline Most favoured & 98 \\
\hline Additional allowed & 2 \\
\hline Generously allowed & 0 \\
\hline Disallowed & 0 \\
\hline \multicolumn{2}{|c|}{$\begin{array}{l}\text { a Friedel's pairs merged. } \\
\text { b } R_{\text {merge }}=\sum_{h k l} \sum_{i}\left|I_{i}(h k l)-\langle I(h k l)\rangle\right| / \sum_{h k l} \sum_{i} I_{i}(h k l) \text {. } \\
\text { c } R \text {-factor }=\sum_{h k l}|| F_{\text {obs }}(h k l)|-k| F_{\text {cal }}(h k l) \| / \sum_{h k l}\left|F_{\text {obs }}(h k l)\right| \text {, in } \\
\text { which } F_{\text {obs }} \text { and } F_{\text {cal are the observed and calculated structure }} \text { are obsections omitted in the refinement } \\
\text { factors, respectively. R-free, the cross-validation } R \text {-factor was } \\
\text { calculated with } 5 \% \text { of the reflections omited } \\
\text { process. }\end{array}$} \\
\hline
\end{tabular}

STAT6 helix is tilted by approximately $50^{\circ}$ relative to the axis of helix $\mathrm{D}_{\alpha}$ of the NCoA-1 PAS-B domain. At the same time the axis of the STAT6 helix is also tilted by about $50^{\circ}$ against the $\beta$-sheet of the NCoA-1 PAS-B domain. Because of the concave shape of the $\beta$-sheet the helical portion of the STAT6 fragment packs against the $B_{\beta}$ strand (Figure 1).

The closest structural similarity to the NCoA-1 PAS-B domain, as calculated by DALI, ${ }^{28}$ was found for photoactive yellow protein (PYP), a bacterial light-sensing protein ${ }^{29}$ and the PAS domain of the HERG (human ether-a-gogo-related gene) voltage-dependent $\mathrm{K}^{+}$-channel. ${ }^{30}$ There is no significant sequence similarity between the NCoA-1 PAS-B domain and the PAS domains of PYP and HERG. Nevertheless their basic topology is very similar (Figure 2(a) and (b)). But there are striking differences in the structural organization of the helical portion. When the whole structures of the PYP and HERG PAS domains are individually superimposed to the NCoA-1 PAS-B domain, the r.m.s.d. values are $3.0 \AA$ and $2.7 \AA$, respectively (alignment of $81 \mathrm{C}_{\alpha}$ atoms of PYP and 73 of HERG with structurally equivalent atoms of NCoA-1 PAS-B). When the helical portion of the proteins is excluded from this calculation, the r.m.s.d. drops to $1.6 \AA$ and $1.4 \AA$ (superposition of $38 \mathrm{C}_{\alpha}$ atoms of PYP and HERG, respectively, to structurally equivalent atoms of NCoA-1 PAS-B domain). In PYP and HERG helix $\alpha \mathrm{A}$ is tilted by almost $90^{\circ}$ relative to the homologous NCoA-1 PAS-B domain helix $\mathrm{C}_{\alpha}$ (Figure 2(c)). This brings the next helix $\alpha B$ of PYP and HERG in close vicinity of the strand $\beta B$. The following "vine", which consists of a long ordered loop and a single turn of $3_{10}$ helix (helix $\alpha^{\prime} \mathrm{A}$ ), ${ }^{30}$ covers the $\beta$-sheet (Figure $2(\mathrm{c})$ ). In contrast, a vine does not exist in the NCoA-1 PAS-B domain. Here helix $D_{\alpha}$, which makes three and a half turns, spans across the whole $\beta$-sheet. It is located much further away from the $\beta$-sheet than helix $\alpha \mathrm{B}$ and its associated vine in PYP and HERG. The closest $\mathrm{C}_{\alpha}$ distances of $\mathrm{D}_{\alpha}$ to $\mathrm{A}_{\beta}$ and $\mathrm{B}_{\beta}$ are $7.8 \AA$ and $8.3 \AA$, respectively. In HERG and PYP the closest $\mathrm{C}_{\alpha}$ distances from the vine to $\beta \mathrm{B}$ are only $4.2 \mathrm{~A}$ and $4.8 \AA$. Thus in NCoA-1 a "binding" groove is formed between $D_{\alpha}$ and $B_{\beta}$ where the STAT6 fragment can bind. In a structural alignment of the HERG PAS domain and the STAT6/NCoA-1 PAS-B complex (Figure 2(d)), the STAT6 peptide occupies the position of the HERG domain helix $\alpha \mathrm{B}$, although in a different orientation.

\section{Interaction of the STAT6 peptide with the NCoA-1 PAS-B domain}

The electron density of the structured STAT6 peptide (Figure 3(a)) allows detailed analysis of its interactions with the NCoA-1 PAS-B domain. The STAT6 peptide binds into a shallow groove at the surface of the NCoA-1 PAS-B domain (Figure 3(b) and (c)). The groove is about $20 \AA$ long and $10 \AA$ wide as estimated from $\mathrm{C}_{\alpha}$ distances. Hydrophobic residues seal the floor of the groove (Ile272, Ile273, Ile275 on the $B_{\beta}$ strand, the methyl group of Thr277 on the $C_{\alpha}$ helix, Trp288, Val292, Ile296 and Phe300 on the $\mathrm{D}_{\alpha}$ helix), while hydrophilic side-chains, lining the "walls" of the groove, are directed outwards into the solvent region (Figure 4(a) and (b)). The STAT6/NCoA-1 PAS-B domain interface buries $865 \AA^{2}$.

The STAT6 peptide is amphipathic, presenting hydrophobic residues at the NCoA- 1 interface and polar or hydrophilic residues at the solvent side. The Leu side-chains of the STAT6 LXXLL signature motif are deeply embedded into the hydrophobic floor of the PAS-B domain. The side-chains of $\mathrm{Leu}^{\prime} 802$ and Leu'805 have the least accessible surface within the peptide: $6 \AA^{2}$ and $20 \AA^{2}$, respectively. Their substitution by alanine abolished binding to NCoA-1 in vitro, recruitment of NCoA-1 and consequently its transactivation potential in cells. $^{22}$ Therefore not only the hydrophobicity of the motif, but also the size of the side-chains is 

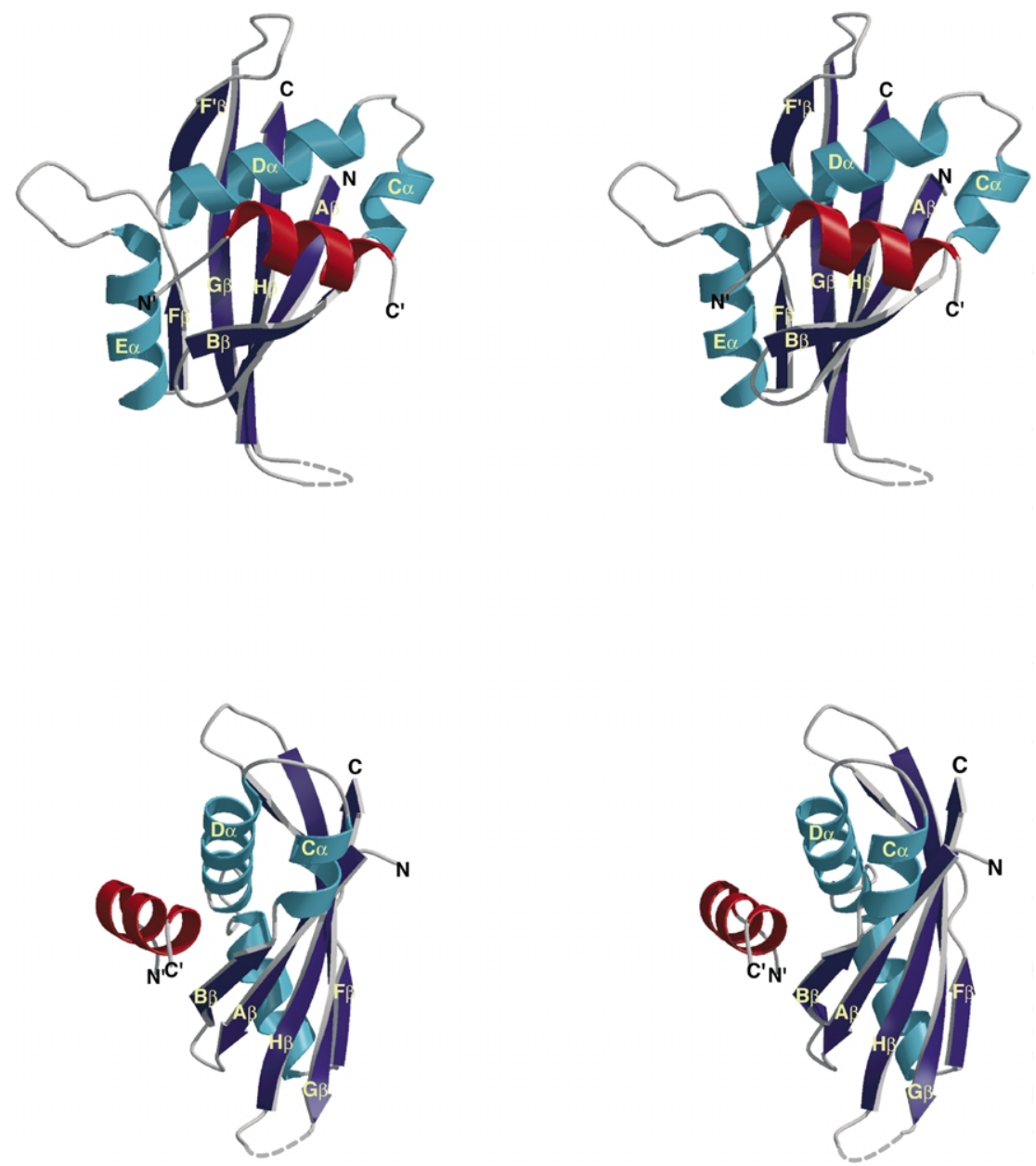

Figure 1. Stereo views of the STAT6/NCoA-1 PAS-B complex in two mutually orthogonal orientations. The $\beta$ sheet and the helices of the PAS-B domain are depicted in dark and light blue, respectively. The STAT6 peptide is shown in red.

essential for binding to the NCoA-1 PAS-B domain. This is clearly explicable by the crystal structure: the molecular surface of the peptide, at the signature-motif, is complementary with the groove in the PAS domain. Leu' 802 fits perfectly into a deep pocket within the hydrophobic groove: it establishes Van-der-Waals interactions with Ile275 and Ile296 (Figure 4(a) and (b)). Leu' 805 fits into a shallower pocket (Figure 4(c)) formed by Ile272 and Ile275 side-chains and by $\mathrm{C}_{\alpha}$ of Ile273 and Ser274 (Figure 4(a)). Also Leu'806 fits into a deep pocket formed by the methyl group of Thr277 and the side-chains of Ile275 and Trp288 (Figure 4(b)). However Leu'806 is more accessible $\left(38 \AA^{2}\right)$ than $\mathrm{Leu}^{\prime} 805$ and $\mathrm{Leu}^{\prime} 802$, because from one side it is not shielded from the solvent.

Pro'796 and Pro'797 in the extended N-terminal part of the STAT6 peptide pack in a shallow depression formed by Phe300 and Ile296 at the Cterminal end of the PAS-B domain $\mathrm{D}_{\alpha}$ helix (Figure 4(a)). The STAT6 peptide is anchored by the Arg293 side-chain through hydrogen bonds with the hydroxyl group of Thr' 803 and the backbone carbonyl of Glu'799. This anchor is strengthened by a weak salt bridge $(3.17 \AA)$ between the side- chains of Arg293 and Glu'799 (Figure 4(b)). The hydrogen bond to the Glu'799 carbonyl is the only direct contact of a PAS domain residue to the backbone of the STAT6 peptide. All other contacts between STAT6 and the PAS domain are sidechain to side-chain interactions. Therefore the peptide does not sit deeply inside the binding cleft, but is rather kept at "arms length", whereby the arms are represented by the Leu side-chains (Figure 4(c)). The spacing between Leu'802 and Leu' 805 is necessary to position these two residues and Leu'806 in their respective pockets and thus allows only one orientation of the peptide in the groove.

The peptide is sitting asymmetrically in the binding cleft of the PAS-B domain. It is tightly packed against the rim containing the Arg293 anchor. Due to this positioning, no ordered water molecules are found between this rim and the peptide. There is a relatively large space between the STAT6 peptide and the opposite rim (Figure 3(b) and (c)). A network of ordered water molecules, which mediate indirect interactions with PAS-B domain residues, fills this space. 


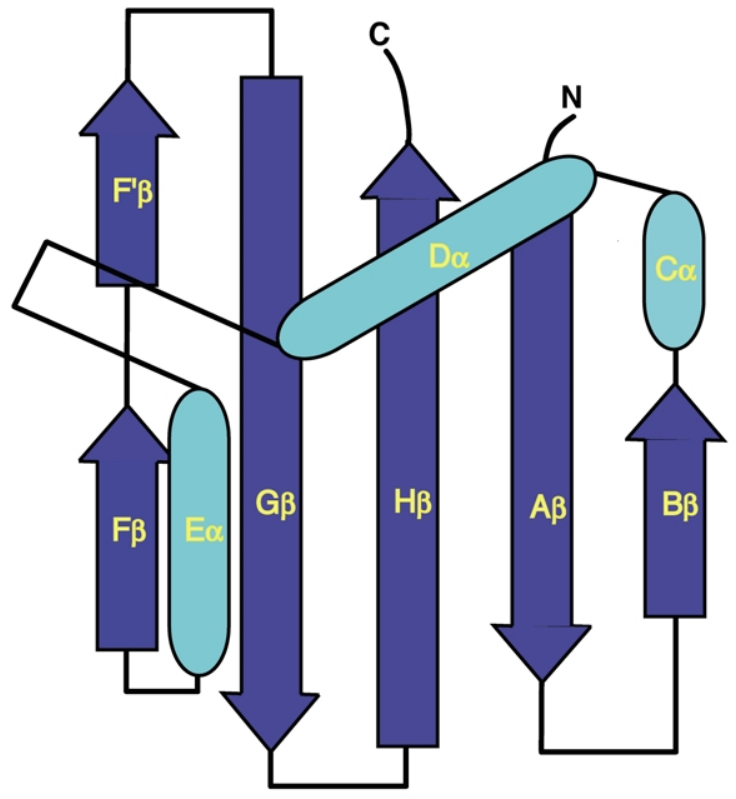

(a)

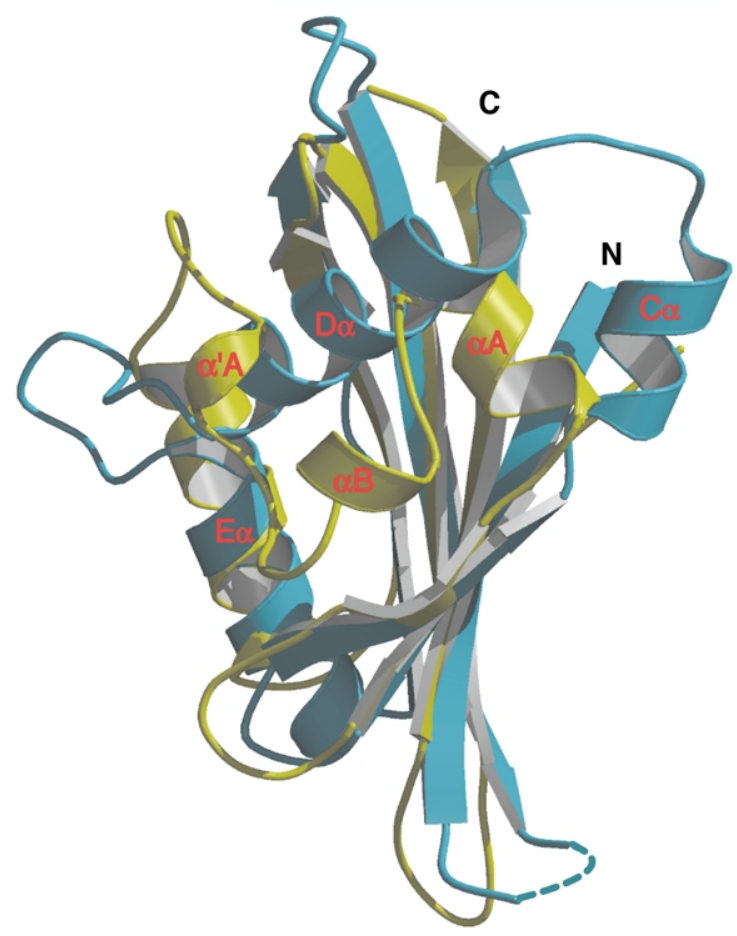

(c)

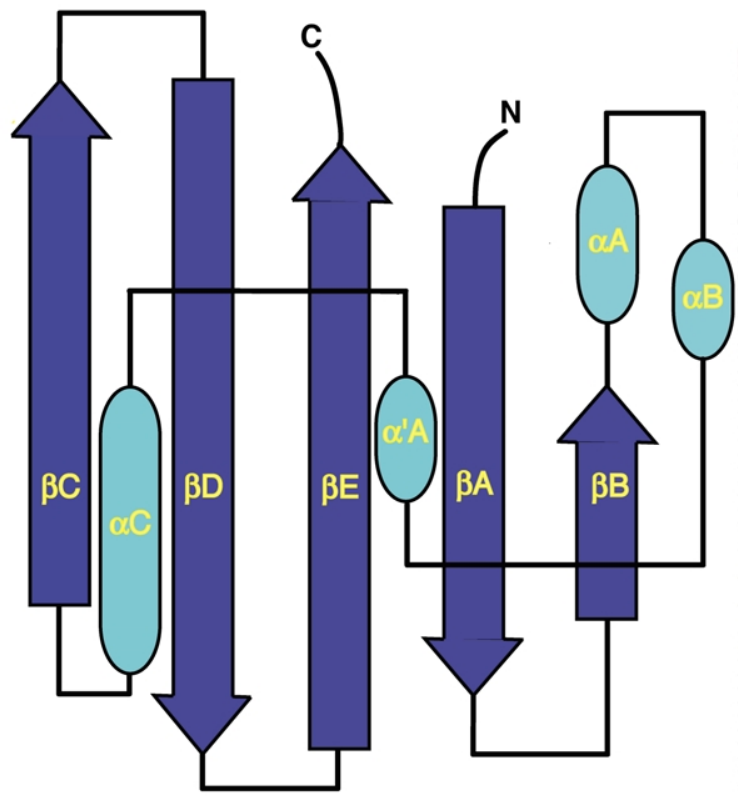

(b)

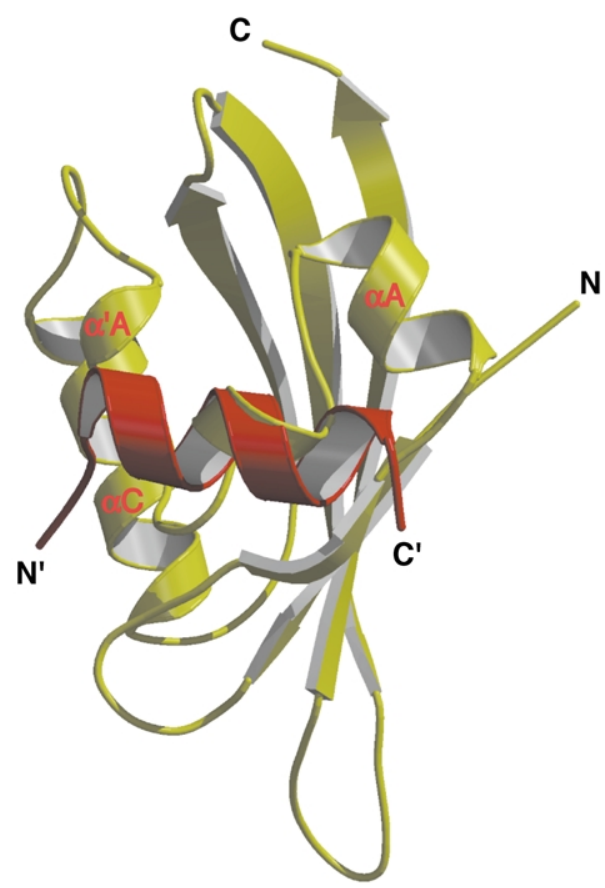

(d)

Figure 2. Topology diagrams of the NCoA-1 PAS-B domain (a) and the PYP PAS domain (b). The color code corresponds to Figure 2. (c, d) Superposition of the STAT6/NCoA-1 PAS-B complex and the HERG PAS domain. The HERG domain is depicted in yellow, the STAT6 peptide is shown in red and the NCoA-1 PAS-B domain in light blue. For reasons of clarity in (c) the model of the STAT6 peptide and in (d) the model of the NCoA-1 PAS-B domain are omitted.

\section{The binding specificity between NCoA-1 and STAT6 resides in the PAS-B domain}

It was shown before, that $\mathrm{NCOA}-1$, but not the related family members $\mathrm{NCOA}-2$ and $\mathrm{NCoA}-3$, interacts with STAT6. ${ }^{14}$ To confirm that the binding specificity depends on the PAS-B domain, the interactions of the in vitro translated homologous PAS-B regions from the three $\mathrm{NCOA}$ isoforms with the STAT6 transactivation domain was investigated in 

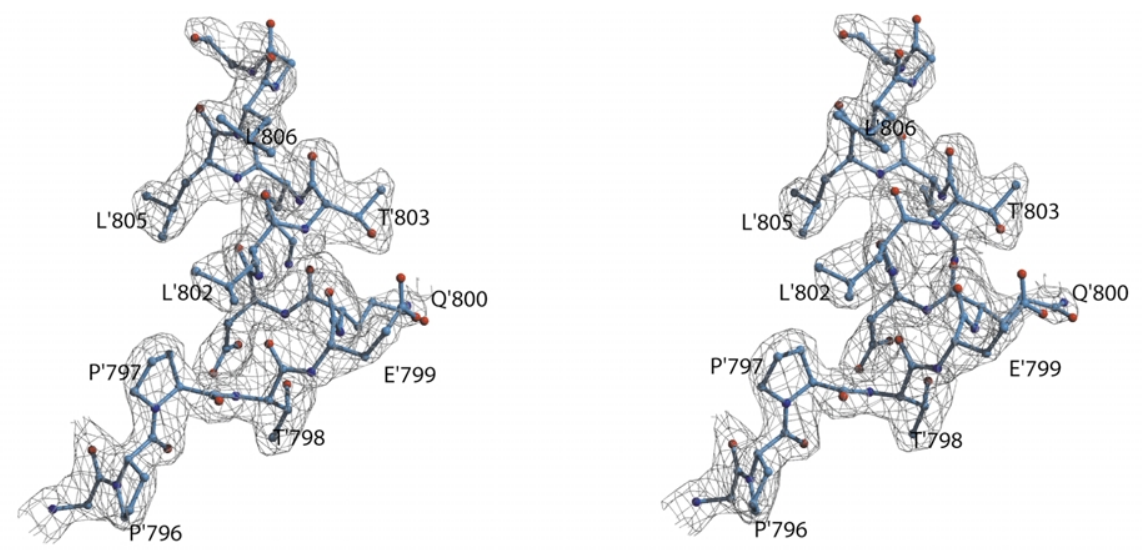

(a)

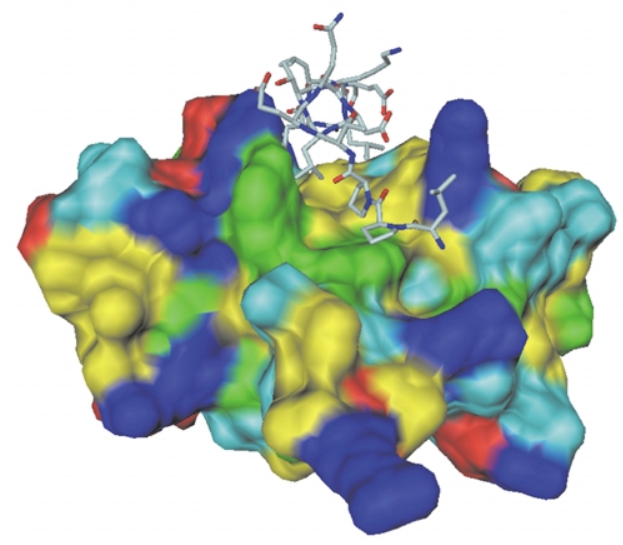

(b)

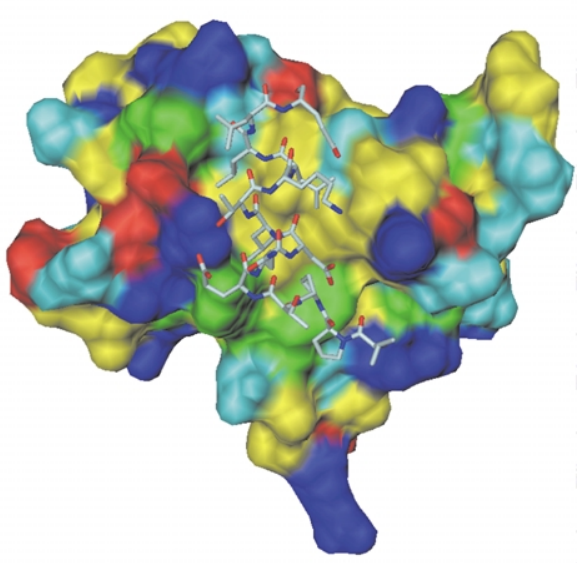

(c)

Figure 3. (a) Model of the STAT6 peptide in the $2 m F_{\mathrm{o}}-D F_{\mathrm{c}}$ electron density map. The map (mesh) is contoured at $0.18 \mathrm{e} / \AA^{3}$. C atoms are in light blue; N, dark blue; and $\mathrm{O}$, red. (b) Surface representation with basic residues colored blue, acidic red, aliphatic yellow, aromatic green and hydrophilic cyan. The bound peptide is shown in a stick representation. View onto the complex with the peptide axis perpendicular to the plane of the Figure. (c) The complex is rotated by $90^{\circ}$ about the horizontal axis of (b). The Figure was generated using the programs MSMS ${ }^{53}$ and DINO $†$.

a GST pulldown experiment (Figure 5(a)). As expected, the NCoA-1 PAS-B domain strongly interacted with the GST fusion protein comprising residues $677-847$ of the STAT6 transactivation domain. In contrast the fragment of NCoA-2 showed only very weak binding and the fragment of NCoA-3 did not interact at all. This indicates that the PAS-B domain alone determines the binding specificity to STAT6.

$\dagger$ Phillippsen, A. (2003). DINO: Visualizing Structural Biology. http://www.dino3d.org

\section{Discussion}

\section{The LXXLL motif is a common module for protein-protein interactions in transcriptional regulation}

Transcriptional activation is mediated by large protein complexes assembled on target gene promoter regions. These complexes contain activators and coactivators of transcription as well as elements of the basal transcription machinery. ${ }^{4}$ The specificity, timing, and degree of transcriptional activation depend not only on the proteins forming the complex, but also on the way they interact with each other. Despite extensive 


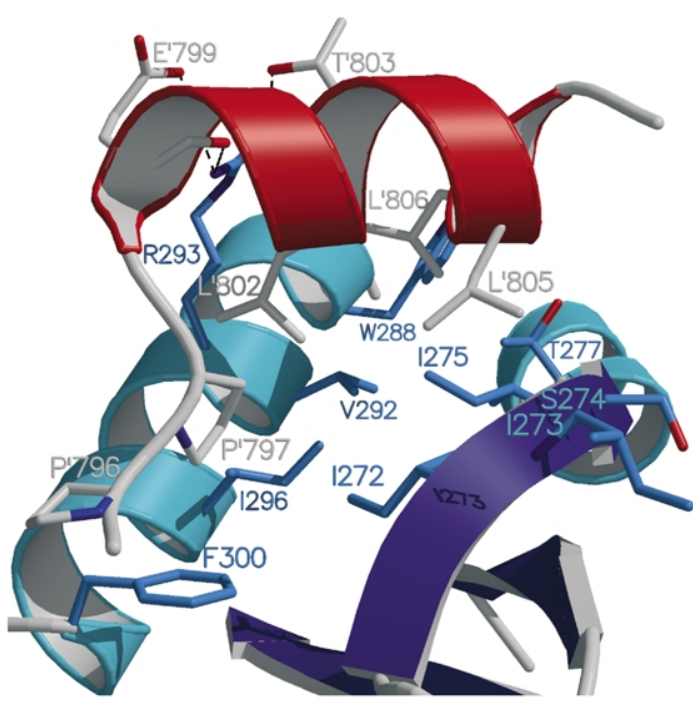

(a)

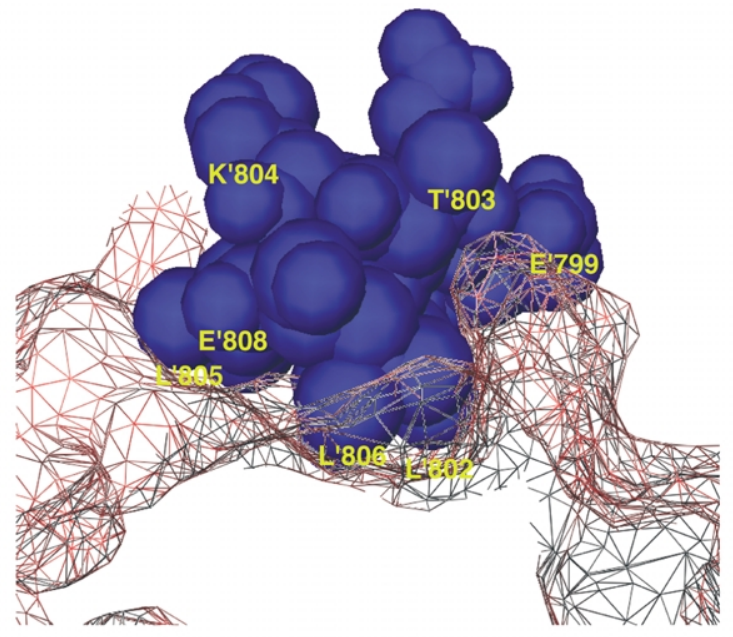

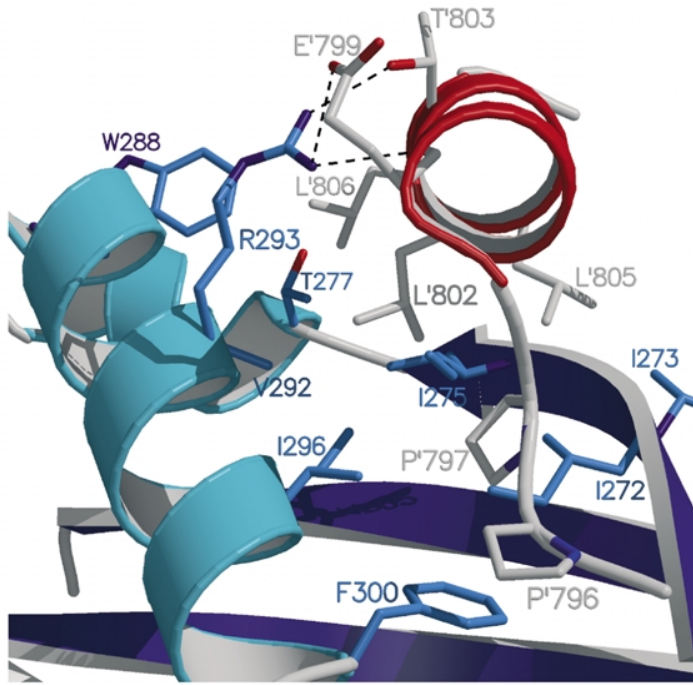

(b)

(c)

Figure 4. Close-ups of the interactions between the STAT6 peptide and the NCoA-1 PAS-B domain. (a) View along the helical axis of the STAT6 peptide. The residues of the STAT6 peptide being involved in interactions are painted in grey. Their partner residues on the NCoA-1 PAS-B domain are painted in blue. Hydrophilic and electrostatic interactions are indicated by dotted lines. (b) The interface in an orientation rotated $90^{\circ}$ about the vertical axis of (a). (c) L'802, L'805 and L'806 tightly fit into the binding groove of the NCoA-1 PAS-B domain. A cross-section of the interface showing the complementarity of the STAT6 peptide with the PAS-B domain groove. The NCoA-1 PAS-B domain surface is represented as a wire mesh and the STAT6 peptide is in space filling representation.

investigation, the molecular basis of the activation function is still poorly understood. The reason is in part because transactivation domains share very little sequence similarity. Moreover they usually have a poor intrinsic propensity to form secondary structure, although they apparently need to make specific interactions with several different target factors. This confirms the notion that the minimal requirement for partner recognition is defined by very short amino acid stretches, which become structured only when bound to target proteins..$^{31-34}$

In the crystal structure of the NCoA-1 PAS-B domain complex with the STAT6(794-814) peptide the ordered part of the peptide (795-807) constitutes such a minimal structural entity. The LXXLL motif has been described before as a module mediating the interaction of the p160/SRC/ NCoA cofactors with nuclear hormone receptors (NRs). ${ }^{35}$ These cofactors contain three LXXLL 
(a)

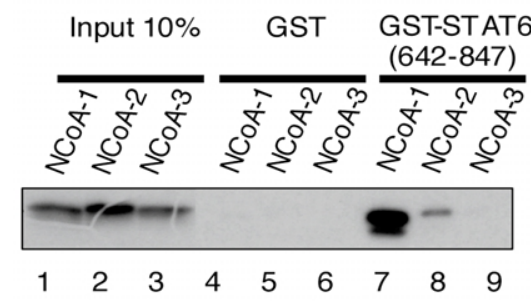

(b)

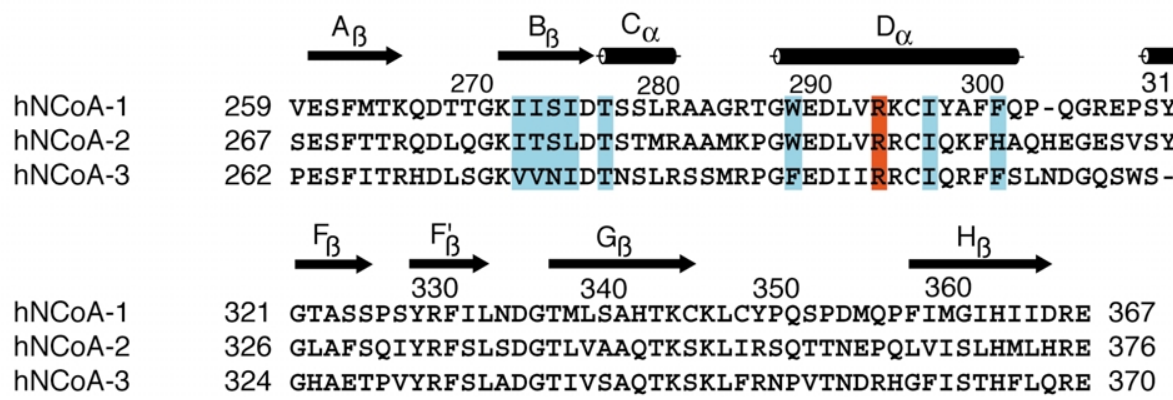

Figure 5. (a) The NCOA-1 PAS-B specifically interacts with the STAT6 transactivation domain. In vitro translated, $\left({ }^{35}\right.$ S)methionine-labeled PAS-B domains of NCoA-1, NCoA-2 and NCoA-3 were incubated with GST or GST STAT6 transactivation domain fusion protein bound to glutathione-Sepharose. Proteins bound to the glutathione-Sepharose were recovered from the beads and analyzed by SDS-PAGE and fluorography (lanes 4-9). Input control in lanes 1-3 reflects $10 \%$ of the total amount of $\left({ }^{35} \mathrm{~S}\right)$ methionine-labeled protein used in the experiments. (b) Sequence alignment of the NCoA-1 fragment 259-367, which comprises the PAS-B domain, with the homologous fragments 267-376 of NCoA-2 and 262-370 of NCoA-3. The arginine anchoring the STAT6 peptide is painted in red. Residues involved in hydrophobic interactions are painted in light blue.

motifs in their so-called nuclear receptor interaction domain (NID). ${ }^{36,37}$ These LXXLL motifs bind to the transcriptional activation 2 function (AF2) in the ligand binding domain (LBD) of nuclear hormone receptors. In addition all SRC/ NCoA coactivators contain LXXLL motifs in a domain that mediates complex formation with the coactivator CBP (CID). The structure of the CID of NCoA-3 in complex with the glutamine rich domain of CBP was recently solved. ${ }^{38}$ It will be interesting to see, if the NCoA PAS-B domain interacts with some of its own LXXLL motifs in a inter- or intra molecular manner. We are currently investigating the specificity of different LXXLL motifs for binding to the PAS domain.

Variants of the LXXLL motif, where leucine can be replaced by other bulky hydrophobic residues, play an essential role in the complex of the p53 transactivation domain with the MDM2 oncoprotein $^{33}$ and in the complex of the kinase inducible transactivation domain (KID) of CREB with the CBP KIX domain. ${ }^{34,38}$ In all available

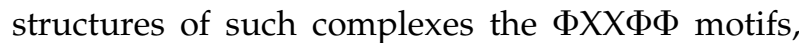
$\Phi$ being bulky hydrophobic residues, form amphipathic helices, whose hydrophobic side-chains tightly interact with their target proteins by formation of a hydrophobic interface. The different target proteins all present a hydrophobic groove, where the $\Phi Х Х \Phi \Phi$ motif binds.
The LXXLL motif of NCoA/SRC cofactors is positioned in the binding groove of the NR AF2 domain by a charge clamp formed by a lysine and a glutamic acid of the AF2 domain. ${ }^{39,40}$ The p53 peptide containing an FXXWL motif has extensive hydrophobic contacts with the MDM2 interaction domain. ${ }^{33}$ But in addition two spatially separated hydrophilic contacts are clearly involved in clamping the peptide into the binding groove. In the KID/KIX and NCoA-3/CBP complex multiple electrostatic interactions and hydrogen bonds along the interface are also essential for positioning the amphipathic helices in their binding grooves. ${ }^{34,38}$

A charge or a hydrophilic clamp is not found in the complex of the STAT6 peptide with the NCoA-1 PAS-B domain. Only the side-chain of PAS-B domain Arg293 forms two hydrogen bonds and a salt bridge with the STAT6 peptide (Figure 4(a) and (b)). Due to the missing clamp, hydrophobic interactions play the central role in positioning the STAT6 fragment in the NCoA-1 PAS-B domainbinding groove. Therefore the STAT6 fragment bound to the NCoA-1 PAS-B domain is the first activator/coactivator complex so far studied where the surface complementarity between hydrophobic faces almost exclusively defines the orientation of the two proteins relative to each other. 


\section{The PAS-B domain is a new interaction module for the LXXLL motif}

While the LXXLL and similar motifs form structurally homologous amphipathic helices, their target proteins share no structural similarity. The binding grooves of the NR AF2 domain, ${ }^{39,40}$ the CBP KIX domain, ${ }^{34}$ the CBP glutamine-rich domain $^{38}$ and the $\mathrm{NH}_{2}$-terminal MDM2 domain ${ }^{33}$ are mostly constituted by different helical folds. The structure of the NCoA-1 PAS-B domain in complex with the STAT6 peptide adds a new fold to this family of $\Phi Х X \Phi \Phi$ motif interacting domains. It extends the number of signal transduction modules, whose structures have been solved in complex with protein fragments containing unmodified signature motifs. ${ }^{41}$

The contribution of the PAS domain in NCoA/ SRC coactivator function is not yet well understood. A potential role in coactivator multiprotein complex formation and in contacting different transcription factors has been suggested. ${ }^{18,19}$ Three-dimensional structures of several prokaryotic and eukaryotic PAS domains have already been solved. ${ }^{29,30,42,43}$ But so far structures of protein-protein complexes mediated by PAS domains have not been obtained. The crystal structure of the NCoA-1 PAS-B domain in complex with the STAT6 LXXLL motif allows now detailed insight into proteinprotein interactions involving a PAS domain. It will be the basis for further studies of this domain in complex with other binding partners, to ultimately obtain a better understanding of the PAS domain function in SRC/NCoA proteins. The STAT6 signaling pathway is an attractive target for the treatment of allergic diseases, because STAT6 is crucial for class switching to IgE, a pivotal factor in the pathogenesis of asthma. The inhibition of the NCoA-1 PAS-B domain/STAT6 LXXLL complex formation by small organic molecules might be an attractive approach to inhibit this pathway. The structure of the complex presented here, will be important to identify and characterize such inhibitors.

\section{Determinants of binding specificity}

A sequence alignment of residues 259-367 of the NCoA-1 PAS-B domain with the homologous sequences 267-376 of NCoA-2 and 262-370 of NCoA-3 (Figure 5(b)) demonstrates, that those residues making specific side-chain contacts to the STAT6 peptide, are all conserved or highly homologous between the three PAS-B domain sequences. Therefore based on the available structure of the STAT6/NCoA-1 PAS-B complex we cannot identify individual residues in the NCoA-1 PAS-B domain, which cause specific binding to the STAT6 LXXLL motif (Figure 5(a)). The inability of NCoA-2 and NCoA-3 to bind to STAT6, despite of the high sequence conservation in their PAS-B regions, strongly suggests that surface complementarity plays an essential role not only for the orientation of the STAT6 peptide in the NCoA-1 binding groove, but also for binding specificity. To test this hypothesis mutagenesis of distinct amino acids that will disturb the surface complementarity is on the way.

\section{Materials and Methods}

\section{Protein expression and purification}

Fragments $257-385$ and $257-420$ of the NCoA-1 PAS-B region were cloned into a modified $\mathrm{pET} 16 \mathrm{~b}$ vector containing a Tobacco-Etch-Virus (TEV) protease cleavage site. The proteins were expressed in E. coli and purified using Ni-NTA-Agarose. Details of the expression and purification will be published elsewhere. Briefly, after removing the His-tag with TEV protease the proteins were dialyzed against $50 \mathrm{mM}$ Hepes, $\mathrm{pH} 7.0,150 \mathrm{mM}$ $\mathrm{NaCl}, 2 \mathrm{mM}$ DTT and concentrated to $0.2 \mathrm{ml}$ volume. For assembly of the complex the STAT6 peptide was added in 1.5 molar excess. The samples were passed through a Superdex $75 \mathrm{HR}$ gel filtration column (Amersham Biosciences) and concentrated to $\sim 19 \mathrm{mg} / \mathrm{ml}$ for crystallization trials.

\section{Crystallization and data collection}

Crystals of NCoA-1 PAS-B domain fragment 257-385 in complex with the STAT6(794-814) peptide were obtained at $20^{\circ} \mathrm{C}$ by the hanging drop vapor diffusion technique using $0.2 \mathrm{M} \mathrm{LiCl}$ and $20 \%$ PEG3350 as precipitant. Crystals were soaked for three minutes in a cryoprotectant solution, which consisted of the mother liquor containing $5 \%$ glycerol and $0.5 \mathrm{M} \mathrm{NaI}$. Data collection was performed at $100 \mathrm{~K}$ using $\mathrm{CuK} \alpha$ radiation $(1.54 \AA)$ generated by a rotating anode generator. Diffraction data to $2.2 \AA$ were collected on a Mar345 (Mar Research Inc.) image plate detector.

Data were processed with DENZO and SCALEPACK. ${ }^{44}$ Data statistics are reported in Table 1.

\section{Structure solution and refinement}

The structure was solved by single anomalous wavelength diffraction, exploiting the anomalous signal of iodide ions, which at the home source $\mathrm{CuK} \alpha$ wavelength $(1.54 \AA)$ is $f^{\prime \prime}=6.8$ electrons. ${ }^{25}$ The anomalous signal-to-noise ratio $(|\Delta F| / \sigma(\Delta F))$ as calculated by XPREP (Bruker-AXS, Madison, USA) was quite strong (Table 1), indicating that the crystal had incorporated the iodide ions during the soak. It was significantly decreasing only below $3.0 \AA$ resolution, which was used as the resolution cutoff to search for iodide ions in the unit cell by SHELXD. ${ }^{45}$ Phase extension and improvement by density modification was performed for both heavy-atom enantiomorphs with SHELXE ${ }^{46}$ and only after ten cycles showed a clear discrimination between them (Table 1). The resulting electron density maps are interpretable: 100 cycles of automatic model tracing, alternated by structure refinement by ARP/wARP ${ }^{47}$ (in a total number of 500 refinement cycles), resulted in modeling 113 residues. Refinement consisted of inspection of electron density maps and manual model building by XtalView, ${ }^{48}$ followed by positional and $\mathrm{B}$ factor restrained refinement with REFMAC ${ }^{49}$ coupled with ARP solvent building. Refinement and map calculation was performed 
with $95 \%$ of the data, $5 \%$ of randomly chosen reflections were used for the calculation of $R_{\text {free }}$. The final model of the complex between NCoA-1 PAS-B domain and STAT6 includes 119 of the 153 residues, 116 water molecules and 5 iodide ions. Figures were prepared with MOLSCRIPT, ${ }^{50}$ BOBSCRIPT, $^{51}$ RASTER3D $^{52}$ MSMS $^{53}$ and DINO $\dagger$.

\section{GST-in vitro binding assays}

Fragments $260-370$ of NCoA-1/SRC1, 268-379 of NCoA-2/hTIF2 and 263-375 of NCoA-3/hAIB1 were cloned into the pET30A vector (Novagene). Recombinant cDNA of these expression vectors were transcribed and translated in vitro in reticulocyte lysate (Promega) in the presence of $\left({ }^{35} \mathrm{~S}\right)$ methionine according to the manufacturer's instructions. GST or GST fusion proteins were expressed in E. coli and purified with glutathione-Sepharose beads (Amersham Biosciences). For binding assays, GST fusions or GST alone $(1.5-5 \mu \mathrm{g})$, bound to glutathione-Sepharose beads, were incubated with labeled proteins in $200 \mu \mathrm{l}$ of binding buffer as described. ${ }^{54}$ After extensive washing bound proteins were eluted and separated on SDS-PAGE gels. Radiolabeled proteins were visualized by fluorography. Amounts and integrity of bound proteins were estimated on SDS-PAGE gels by Coomassie staining.

\section{Coordinates}

The coordinates and the structure factors have been deposited in the Protein Data Bank (accession code 1OJ5).

\section{Acknowledgements}

We thank Kerstin Overkamp for peptide synthesis, Uwe Pleßmann and Dr Henning Urlaub for mass spectrometry and Dr Jochen Junker for computing assistance. We are grateful to Dr Thomas Schneider and Prof. George Sheldrick for support and advice during data collection. A.R. and S.B. were supported by the Max-Planck-Gesellschaft and Deutsche Forschungsgemeinschaft (grant Be 2345).

\section{References}

1. Darnell, J. E., Jr (1997). STATs and gene regulation. Science, 277, 1630-1635.

2. Darnell, J. E., Jr, Kerr, I. M. \& Stark, G. R. (1994). JakSTAT pathways and transcriptional activation in response to IFNs and other extracellular signaling proteins. Science, 264, 1415-1421.

3. Ihle, J. N. (1996). STATs: signal transducers and activators of transcription. Cell, 84, 331-334.

4. Lemon, B. \& Tjian, R. (2000). Orchestrated response: a symphony of transcription factors for gene control. Genes Dev. 14, 2551-2569.

5. Smerz-Bertling, C. \& Duschl, A. (1995). Both interleukin 4 and interleukin 13 induce tyrosine phosphoryl-

$\dagger$ Phillippsen, A. (2003). DINO: Visualizing Structural Biology. http://www.dino3d.org ation of the 140-kDa subunit of the interleukin 4 receptor. J. Biol. Chem. 270, 966-970.

6. Shimoda, K., van Deursen, J., Sangster, M. Y., Sarawar, S. R., Carson, R. T., Tripp, R. A. et al. (1996). Lack of IL-4-induced Th2 response and IgE class switching in mice with disrupted Stat6 gene. Nature, 380, 630-633.

7. Kaplan, M. H., Wurster, A. L., Smiley, S. T. \& Grusby, M. J. (1999). Stat6-dependent and -independent pathways for IL-4 production. J. Immunol. 163, 6536-6540.

8. Hoey, T. \& Schindler, U. (1998). STAT structure and function in signaling. Curr. Opin. Genet. Dev. 8, 582-587.

9. Becker, S., Groner, B. \& Muller, C. W. (1998). Threedimensional structure of the Stat3beta homodimer bound to DNA. Nature, 394, 145-151.

10. Chen, X., Vinkemeier, U., Zhao, Y., Jeruzalmi, D., Darnell, J. E., Jr \& Kuriyan, J. (1998). Crystal structure of a tyrosine phosphorylated STAT-1 dimer bound to DNA. Cell, 93, 827-839.

11. Lu, B., Reichel, M., Fisher, D. A., Smith, J. F. \& Rothman, P. (1997). Identification of a STAT6 domain required for IL-4-induced activation of transcription. J. Immunol. 159, 1255-1264.

12. Moriggl, R., Berchtold, S., Friedrich, K., Standke, G. J., Kammer, W., Heim, M. et al. (1997). Comparison of the transactivation domains of Stat5 and Stat6 in lymphoid cells and mammary epithelial cells. Mol. Cell. Biol. 17, 3663-3678.

13. Goenka, S., Youn, J., Dzurek, L. M., Schindler, U., YuLee, L. Y. \& Boothby, M. (1999). Paired Stat6 C-terminal transcription activation domains required both for inhibition of an IFN-responsive promoter and trans-activation. J. Immunol. 163, 4663-4672.

14. Litterst, C. M. \& Pfitzner, E. (2001). Transcriptional activation by STAT6 requires the direct interaction with NCoA-1. J. Biol. Chem. 276, 45713-45721.

15. Gingras, S., Simard, J., Groner, B. \& Pfitzner, E. (1999). p300/CBP is required for transcriptional induction by interleukin- 4 and interacts with Stat6. Nucl. Acids Res. 27, 2722-2729.

16. Goodman, R. H. \& Smolik, S. (2000). CBP/p300 in cell growth, transformation, and development. Genes Dev. 14, 1553-1577.

17. Vo, N. \& Goodman, R. H. (2001). CREB-binding protein and p300 in transcriptional regulation. J. Biol. Chem. 276, 13505-13508.

18. Leo, C. \& Chen, J. D. (2000). The SRC family of nuclear receptor coactivators. Gene, 245, 1-11.

19. Liao, L., Kuang, S. Q., Yuan, Y., Gonzalez, S. M., O'Malley, B. W. \& Xu, J. (2002). Molecular structure and biological function of the cancer-amplified nuclear receptor coactivator SRC-3/AIB1. J. Steroid Biochem. Mol. Biol. 83, 3-14.

20. Korzus, E., Torchia, J., Rose, D. W., Xu, L., Kurokawa, R., McInerney, E. M. et al. (1998). Transcription factorspecific requirements for coactivators and their acetyltransferase functions. Science, 279, 703-707.

21. Kurokawa, R., Kalafus, D., Ogliastro, M. H., Kioussi, C., Xu, L., Torchia, J. et al. (1998). Differential use of CREB binding protein-coactivator complexes. Science, 279, 700-703.

22. Litterst, C. M. \& Pfitzner, E. (2002). An LXXLL motif in the transactivation domain of STAT6 mediates recruitment of NCoA-1/SRC-1. J. Biol. Chem. 277, 36052-36060.

23. Yao, T. P., Ku, G., Zhou, N., Scully, R. \& Livingston, D. M. (1996). The nuclear hormone receptor coactivator SRC-1 is a specific target of p300. Proc. Natl Acad. Sci. USA, 93, 10626-10631. 
24. Geourjon, C. \& Deleage, G. (1995). SOPMA: significant improvements in protein secondary structure prediction by consensus prediction from multiple alignments. Comput. Appl. Biosci. 11, 681-684.

25. Dauter, Z. \& Dauter, M. (2001). Entering a new phase: using solvent halide ions in protein structure determination. Structure (Camb), 9, R21-R26.

26. Laskowski, R. A., Macarthur, M. W., Moss, D. S. \& Thornton, J. M. (1993). Procheck-a program to check the stereochemical quality of protein structures. J. Appl. Crystallog. 26, 283-291.

27. Cruickshank, D. W. (1999). Remarks about protein structure precision. Acta Crystallog. D Biol. Crystallog. 55 (Pt 3), 583-601.

28. Holm, L. \& Sander, C. (1993). Protein structure comparison by alignment of distance matrices. J. Mol. Biol. 233, 123-138.

29. Genick, U. K., Soltis, S. M., Kuhn, P., Canestrelli, I. L. \& Getzoff, E. D. (1998). Structure at $0.85 \AA$ resolution of an early protein photocycle intermediate. Nature, 392, 206-209.

30. Morais Cabral, J. H., Lee, A., Cohen, S. L., Chait, B. T., Li, M. \& Mackinnon, R. (1998). Crystal structure and functional analysis of the HERG potassium channel $\mathrm{N}$ terminus: a eukaryotic PAS domain. Cell, 95, 649-655.

31. Ptashne, M. \& Gann, A. (1997). Transcriptional activation by recruitment. Nature, 386, 569-577.

32. Uesugi, M., Nyanguile, O., Lu, H., Levine, A. J. \& Verdine, G. L. (1997). Induced alpha helix in the VP16 activation domain upon binding to a human TAF. Science, 277, 1310-1313.

33. Kussie, P. H., Gorina, S., Marechal, V., Elenbaas, B., Moreau, J., Levine, A. J. \& Pavletich, N. P. (1996). Structure of the MDM2 oncoprotein bound to the p53 tumor suppressor transactivation domain. Science, 274, 948-953.

34. Radhakrishnan, I., Perez-Alvarado, G. C., Parker, D., Dyson, H. J., Montminy, M. R. \& Wright, P. E. (1997). Solution structure of the KIX domain of CBP bound to the transactivation domain of CREB: a model for activator:coactivator interactions. Cell, 91, $741-752$.

35. Heery, D. M., Kalkhoven, E., Hoare, S. \& Parker, M. G. (1997). A signature motif in transcriptional coactivators mediates binding to nuclear receptors. Nature, 387, 733-736.

36. Moras, D. \& Gronemeyer, H. (1998). The nuclear receptor ligand-binding domain: structure and function. Curr. Opin. Cell. Biol. 10, 384-391.

37. Darimont, B. D., Wagner, R. L., Apriletti, J. W., Stallcup, M. R., Kushner, P. J., Baxter, J. D. et al. (1998). Structure and specificity of nuclear receptor-coactivator interactions. Genes Dev. 12, 3343-3356.

38. Demarest, S. J., Martinez-Yamout, M., Chung, J., Chen, H., Xu, W., Dyson, H. J. et al. (2002). Mutual synergistic folding in recruitment of $\mathrm{CBP} / \mathrm{p} 300$ by p160 nuclear receptor coactivators. Nature, 415, 549-553.

39. Nolte, R. T., Wisely, G. B., Westin, S., Cobb, J. E., Lambert, M. H., Kurokawa, R. et al. (1998). Ligand binding and co-activator assembly of the peroxisome proliferator-activated receptor-gamma. Nature, 395, 137-143.

40. Bledsoe, R. K., Montana, V. G., Stanley, T. B., Delves, C. J., Apolito, C. J., McKee, D. D. et al. (2002). Crystal structure of the glucocorticoid receptor ligand binding domain reveals a novel mode of receptor dimerization and coactivator recognition. Cell, 110, 93-105.
41. Pawson, T. \& Nash, P. (2003). Assembly of cell regulatory systems through protein interaction domains. Science, 300, 445-452.

42. Amezcua, C. A., Harper, S. M., Rutter, J. \& Gardner, K. H. (2002). Structure and interactions of PAS kinase N-terminal PAS domain: model for intramolecular kinase regulation. Structure (Camb), 10, 1349-1361.

43. Vreede, J., van der Horst, M. A., Hellingwerf, K. J., Crielaard, W. \& van Aalten, D. M. (2003). PAS domains. Common structure and common flexibility. J. Biol. Chem. 278, 18434-18439.

44. Otwinowski, Z. \& Minor, W. (1997). Processing of Xray diffraction data collected in oscillation mode. Macromolecular Crystallography, Pt A, vol. 276. pp. 307-326.

45. Schneider, T. R. \& Sheldrick, G. M. (2002). Substructure solution with SHELXD. Acta Crystallog. D Biol. Crystallog. 58, 1772-1779.

46. Sheldrick, G. M. (2002). Macromolecular phasing with SHELXE. Z. Kristallogr. 217, 644-650.

47. Perrakis, A., Morris, R. \& Lamzin, V. S. (1999). Automated protein model building combined with iterative structure refinement. Nature Struct. Biol. 6, 458-463.

48. McRee, D. E. (1999). XtalView Xfit-a versatile program for manipulating atomic coordinates and electron density. J. Struct. Biol. 125, 156-165.

49. Murshudov, G. N., Vagin, A. A. \& Dodson, E. J. (1997). Refinement of macromolecular structures by the maximum-likelihood method. Acta Crystallog. sect. D-Biol. Crystallog. 53, 240-255.

50. Kraulis, P. J. (1991). Molscript-a program to produce both detailed and schematic plots of protein structures. J. Appl. Crystallog. 24, 946-950.

51. Esnouf, R. M. (1999). Further additions to MolScript version 1.4, including reading and contouring of electron-density maps. Acta Crystallog. D Biol. Crystallog. 55 (Pt 4), 938-940.

52. Merritt, E. A. \& Bacon, D. J. (1997). Raster3D: photorealistic molecular graphics. Macromolecular Crystallography, Pt B, vol. 277. pp. 505-524.

53. Sanner, M. F., Olson, A. J. \& Spehner, J. C. (1996). Reduced surface: an efficient way to compute molecular surfaces. Biopolymers, 38, 305-320.

54. Pfitzner, E., Jahne, R., Wissler, M., Stoecklin, E. \& Groner, B. (1998). p300/CREB-binding protein enhances the prolactin-mediated transcriptional induction through direct interaction with the transactivation domain of Stat5, but does not participate in the Stat5-mediated suppression of the glucocorticoid response. Mol. Endocrinol. 12, 1582-1593.

Edited by P. Wright

(Received 28 August 2003; received in revised form 10 December 2003; accepted 12 December 2003)

$$
\begin{aligned}
& \text { science } \boldsymbol{d} \text { directo } \\
& \text { wwW.sciencedirect.com }
\end{aligned}
$$

Supplementary Material for this paper comprising one Figure is available on Science Direct 